\title{
КЛИНИЧЕСКАЯ НАРКОЛОГИЯ
}

\author{
УДК 616.89-008.441.12:575.174.015.3
}

Для цитирования: Николишин А.Е., Бродянский В.М., Чупрова Н.А., Сулимов Г.Ю., Кибитов А.О. В поисках наследственной формы алкогольной зависимости: типология по Клонинджеру, динамика формирования синдрома отмены, семейная отягощенность и оценка генетического риска. Сибирский вестник психиатрии и наркологии. 2018; 2 (99): 82-88. https://doi.org/10.26617/1810-3111-2018-2(99)-82-88

\section{В поисках наследственной формы алкогольной зависимости: типология по Клонинджеру, динамика формирования синдрома отмены, семейная отягощенность и оценка генетического риска}

\author{
Николишин А.Е. ${ }^{1}$, Бродянский В.М. ${ }^{1}$, Чупрова Н.А. ${ }^{1}$, Сулимов Г.Ю. ${ }^{2}$, Кибитов А.О. ${ }^{1}$ \\ ${ }^{1}$ Национальный медицинский исследовательский изентр психиатрии и наркологии имени В.П. Сербского \\ Россия, 119991, Москва, Кропоткинский пер., 23 \\ ${ }^{2}$ Научно-исследовательский кластер АО «Врачи» \\ Россия, 125367, Москва, ул. Габричевского, 5
}

\section{PEЗЮME}

Цель: сравнительный анализ пациентов I и II типа алкогольной зависимости (АЗ) по Клонинджеру с применением количественных оценок семейной отягощенности $(\mathrm{CO})$, клинических характеристик развития и течения АЗ, уровня индивидуального генетического риска развития АЗ по данным генопрофилирования. Материал и методы. 464 стационарных пациента, средний

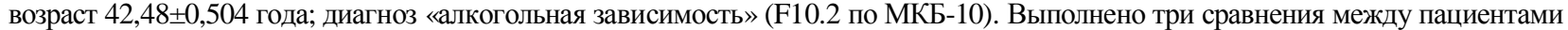
с I и II типами АЗ: в общей группе пациентов, в группе пациентов мужского пола и женского пола. Результаты. В общей группе сравнения у пациентов с II типом АЗ синдром отмены алкоголя (COA) формируется дольше, чем у пациентов с I типом, несмотря на более ранний возраст формирования СОА, а тяга к алкоголю выше как при поступлении, так и при выписке. СО выше у пациентов с II типом АЗ. У пациентов мужского пола с II типом АЗ СОА формируется раньше, тяга к алкоголю достоверно выше как при поступлении, так и при выписке, чем у пациентов с I типом. СО выше у пациентов мужского пола с II типом АЗ. У пациентов женского пола с II типом АЗ СОА формируется дольше, чем у пациентов с I типом, несмотря на более ранний возраст формирования СОА, выраженность тяги к алкоголю и СО достоверно не различается. СО не отличается у женщин с I и II типами АЗ. Уровень генетического риска не отличается у пациентов I и II типов при всех сравнениях. Заключение. АЗ II типа по Клонинджеру с ранним началом злоупотребления встречается в 2 раза чаще у мужчин, чем у женщин, однако около $40 \%$ женщин относятся к этому типу. По результатам исследования II типу свойственны более длительный срок формирования СОА, лучшая динамика уменьшения тяги к алкоголю за время госпитализации при её большей выраженности при поступлении, высокая доля пациентов с СО, особенно её высокой степени, большее число случаев алкоголизма в семье. Это позволяет предположить, что II тип имеет высокий вклад наследственных факторов в риск развития и клинические проявления заболевания.

Ключевые слова: алкогольная зависимость, генетика, наследственность, синдром отмены, семейная отягощенность, генетический риск, полиморфизм генов, дофамин.

\section{ВВЕДЕНИЕ}

Алкогольная зависимость (АЗ) является заболеванием с наследственным предрасположением, вклад генетических факторов в этиопатогенез заболевания значителен и оценивается в 40-70\% [1, 12, 14, 15, 17]. Актуален вопрос о существовании «наследственных» форм АЗ, которые выделены для многих других болезней наследственного предрасположения. Для них характерны раннее начало заболевания, тяжелое течение и большой вклад наследственности в виде семейной отягощенности $(\mathrm{CO})$.

Предрасположенность к алкогольной зависимости - генетически детерминированный и латентно существующий в популяции комплекс особенностей нейрохимических систем мозга, обеспечивающий при употреблении алкоголя быстрое развитие зависимости [7]. Предрасположенность разной степени (от минимальной до максимальной) имеет каждый индивидуум в популяции. Наиболее явным и клинически доступным признаком, позволяющим предпо- лагать наличие значительной генетической предрасположенности у индивидуума, является семейная отягощенность - наличие среди кровных родственников больного случаев этого же заболевания [8] и ее количественная оценка - степень или плотность семейной отягощенности [16]. Количественные оценки СО в виде степени (или плотности) и оценки количества случаев АЗ в семье пациента позволяют оценить не только факт наличия отягощенности, но и измерить «тяжесть» генетического груза конкретного пациента $[4,5,10]$.

Семейная отягощенность и её степень выступают проявлениями генетического фактора этиопатогенеза. Они влияют на функции центральной нервной системы, психофизиологические особенности, черты личности и социальную сферу индивидуума. В результате этих влияний увеличивается генетический риск развития и формируются специфические клинические характеристики течения наркологических заболеваний [5]. 
Генетический риск - вероятность развития заболевания, обусловленная только генетическими причинами. Уровень риска - категориальная (ранговая) оценка - отражает генетически заданную индивидуальную вероятность развития заболевания [5]. Генетический риск развития АЗ или вклад различных вариантов структуры генов (полиморфных локусов) в формирование заболевания можно оценить путем генопрофилирования, проводимого в рамках ДНКдиагностики [7]. Генетические факторы существенно влияют как на риск возникновения А3, так и на клинические проявления заболевания [7, 9, 11].

К. Клонинджер на основе многолетних исследований 862 пациентов с алкогольной зависимостью в Швеции в 1981 г. предложил свою классификацию алкогольной зависимости по возрасту начала злоупотребления алкоголем [13]. Согласно этой типологии, выделяется два типа АЗ, один из которых (тип II) - условно биологический или наследственный тип с ранним началом злоупотребления алкоголем до 25 лет, наличием СО по АЗ, чаще представленный у мужчин, имеет значительно больший вклад генетических факторов, чем I тип (условно «социальный») с началом злоупотребления алкоголем после 25 лет, генетическими и средовыми факторами развития $\mathrm{A} 3$, более частыми расстройствами настроения, например, тревогой или чувством вины. Именно среди пациентов с II типом, вероятно, может быть обнаружен наследственный вариант АЗ Несмотря на то что эта классификация актуальна и используется в настоящее время, в том числе в генетических исследованиях, она критикуется за упрощенный подход. Известно, что многие пациенты II типа не обнаруживают СО и, напротив, многие пациенты I типа имеют CO.

Синдром отмены алкоголя (СОА) является одним из основных проявлений алкогольной зависимости и позволяет диагностировать ее вторую стадию [2]. СОА является очень частой причиной госпитализации больных АЗ в стационар [3].

Учитывая конфликтность и разнообразие данных современных исследований, очевидна необходимость поиска наследственной формы алкогольной зависимости, ее влияния на формирование и развитие алкогольной зависимости с позиций доказательного подхода с использованием количественных методов оценки семейной отягощенности, визуально-аналоговой шкалы тяги к алкоголю и уровня генетического риска с учетом типологии алкогольной зависимости по Клонинджеру.

Цель настоящего исследования - провести сравнительный анализ пациентов I и II типов АЗ по Клонинджеру с использованием количественных оценок $\mathrm{CO}$, клинических характеристик развития и течения А3, а также уровня индивидуального генетического риска развития АЗ по данным генопрофилирования.

\section{МАТЕРИАЛЫ И МЕТОДЫ}

В исследование вошли 448 стационарных пациентов клиники ННЦ наркологии - филиала ФГБУ «НМИЦ ПН им. В.П. Сербского» Минздрава России с диагнозом «алкогольная зависимость» (F10.2 по МКБ-10). Все больные (средний возраст (mean $\pm \mathrm{SE}$ ) $42,58 \pm 0,505$ года) были этнические русские, не родственные между собой, из них 106 женщин (24\%) и 342 мужчины (76\%). Пациенты с диагнозами «органические, включая симптоматические, психические расстройства» (F00-F09), «шизофрения, шизотипические и бредовые расстройства» (F20-F29), «расстройства настроения» (F30-F39) не включались в исследование. Все пациенты подписали информированное согласие на участие в исследовании, исследование проведено с разрешения локального этического комитета ННЦН - филиала ФГБУ «НМИЦ ПН им. В.П. Сербского» Минздрава России.

С использованием клинического анкетирования и клинико-катамнестического метода изучали данные о развитии и течении заболевания: возраст формирования СОА, срок формирования СОА от возраста начала злоупотребления алкоголем.

Тягу к алкоголю в момент поступления и выписки оценивали с помощью визуально-аналоговой шкалы (ВАШ). Пациенту предлагали поставить отметку на неразмеченном отрезке длиной 100 мм с отметками 0 (отсутствие тяги к алкоголю) в начале и 100 (максимальный уровень тяги к алкоголю) в конце отрезка в той точке, которая соответствует его интенсивности тяги к алкоголю. С помощью линейки измеряли расстояние (мм) между 0 (отсутствие тяги к алкоголю) и 100 (максимальный уровень тяги к алкоголю), обеспечивая диапазон оценок от 0 до 100. Более высокий балл указывает на большую интенсивность тяги к алкоголю.

Оценка семейной отягощенности. Информацию о семейной отягощенности по наркологическим и психическим заболеваниям получали путем клинического интервью пациента и близкого родственника (чаще матери). Оценивали факт наличия семейной отягощенности, сумму случаев $\mathrm{CO}$, как сумму случаев заболевания алкогольной зависимостью среди кровных родственников, а также ее степень: средняя степень - один кровный родственник, больной алкогольной зависимостью, высокая степень два и более кровных родственника, больных алкогольной зависимостью.

Оценка генетического риска. У пациентов оценивали индивидуальный уровень генетического риска развития болезней зависимости от психоактивных веществ путем генопрофилирования $[5,6]$. Образцы ДНК, полученные из венозной крови, генотипировали методом полимеразной цепной реакции (ПЦР) с последующим рестрикционным анализом. Были использованы маркеры генетического риска (табл. 1).

По результатам генотипирования оценивали итоговый (результирующий) уровень генетического риска у каждого пациента в баллах: средний - до 1,5 баллов включительно, высокий - 2 и 2,5 балла и крайне высокий -3 и более баллов (табл. 2):

1) оценка общего генетического риска по базовым генетическим маркерам (DRD2_Taq; 
DRD2_Nco - в гене дофаминового рецептора типа 2; HUMTH01 - в гене тирозингидроксилазы). При выявлении определенных вариантов генов начисляли 1 балл в оценке итогового уровня риска, т.е. по 1 баллу за каждый из генотипов: DRD2 TaqI A1/A2, DRD2 NcoI (N1/N1 при наличии DRD2 TaqI A1/A2), HUMTH01 - генотипы 6/6, 7/9, 8/10).

2) оценка специфического риска по аллелям в гене дофаминового рецептора типа 4 (DRD4 48 VNTR и DRD4 120 VNTR). При выявлении следующих аллелей начисляли по 0,5 балла в оценке итогового уровня риска (локус DRD4 VNTR120 S; DRD4 VNTR48 A2, A4, A7, A8).

Баллы отражают статистически достоверную вероятность развития заболевания у носителей генетических маркеров.

Т а б ли ц а 1

\begin{tabular}{|c|c|c|c|c|c|c|}
\hline \multicolumn{7}{|c|}{ Маркеры генетического риска } \\
\hline Продукт гена & Код в тексте & Тип & Аллели (количество) & \begin{tabular}{|c|} 
Аминокислотные \\
замены в белке \\
\end{tabular} & $\begin{array}{c}\text { Локализация } \\
\text { в гене }\end{array}$ & ID dbSNP \\
\hline \multirow{2}{*}{$\begin{array}{l}\text { Дофаминовый рецептор } \\
\text { тип } 2 \text { (DRD2) }\end{array}$} & DRD2_Taq & SNV & G/A (rev: C/T) & Glu713Lys & Экзон 8 & Rs1800497 \\
\hline & DRD2_Nco & SNV & $\mathrm{C} / \mathrm{T}$ & His313= & Экзон 7 & Rs6275 \\
\hline \multirow{2}{*}{$\begin{array}{l}\text { Дофаминовый рецептор } \\
\text { тип } 4 \text { (DRD4) }\end{array}$} & DRD4 120 & VNTR & insDel 120 п.н. (1-2) & & 5'-utr & \\
\hline & DRD4 48 & VNTR & Повторы 48 п.н. (2-11) & & Экзон 3 & Rs778199591 \\
\hline $\begin{array}{l}\text { Фермент тирозингидрок- } \\
\text { силаза (ТН) }\end{array}$ & HUMTH01 & VNTR & TCAT/AATG (от 3 до 14) & & Интрон 1 & \\
\hline
\end{tabular}

Т а б ли ц а 2

\begin{tabular}{l} 
Оценка генетического риска \\
\multicolumn{1}{|c|}{ Оценка генетического риска } \\
\begin{tabular}{|l|l|}
\hline \multicolumn{2}{|c|}{} \\
\hline $\mathrm{R}$ - общий генетический риск развития зависимости от ПАВ \\
\hline $\mathrm{R} 1$ - специфический генетический риск алкоголизма \\
\hline $\mathrm{R} 2$ - специфический генетический риск опийной наркомании \\
\hline $\mathrm{RT}$ - итоговый (результирующий) балл риска) \\
\hline Расчет итогового балла риска как суммы: баллов общего риска и половина баллов специфического риска: $\mathrm{RT}=\mathrm{R}+0,5 *(\mathrm{R} 1+\mathrm{R} 2)$, \\
\hline Оценка уровня риска (RT) \\
\hline Средний & Баллы \\
\hline Высокий & до 1,5 \\
\hline
\end{tabular} \\
\hline
\end{tabular}

Типология алкогольной зависимости по Клонинджеру. У пациентов оценивали тип алкогольной зависимости по Клонинджеру. Были сформированы группы сравнения по возрасту начала систематического злоупотребления алкоголем: I тип - после 25 лет $(\mathrm{n}=197)$, II тип - до 25 лет включительно $(\mathrm{n}=251)$.

Дизайн. Проводили сравнение групп пациентов I и II типов АЗ по Клонинджеру. Второе сравнение проводили в группе пациентов мужского пола. Третье сравнение проводили в группе пациентов женского пола. Сравнения проводили с использованием статистической обработки. Выявляли возможные различия в формировании и течении алкогольной зависимости у пациентов с различной степенью семейной отягощённости и с разным уровнем генетического риска.

Статистическая обработка. Описательная статистика для каждого из клинических параметров и данные качественного и количественного анализов получены с использованием статистического пакета IBM SPSS Statistics 23. Для статистической обработки качественные переменные оценивали с помощью критерия согласия $\chi^{2}$ (Пирсона) с использованием поправки Бонферрони для множественных сравнений. Количественные переменные оценивали с помощью непараметрического U-критерия МаннаУитни, в связи с тем, что ни одна переменная не соответствовала нормальному распределению при проверке с использованием критерия КолмогороваСмирнова $(\mathrm{p} \leq 0,001)$. Статистически значимыми считали различия при $\mathrm{p}<0,05$.

\section{РЕЗУЛЬТАТЫ И ОБСУЖДЕНИЕ}

I. Сравнение групп пащчентов с I и II типами алкогольной зависимости

Пациенты с I и II типами АЗ сравнивались по данным о развитии и течении АЗ. В нашей выборке 197 пациентов (44\%) соответствовали I типу, 251 пациент (56\%) - II типу. Среди пациентов с II типом было достоверно меньше женщин $(\mathrm{n}=40 ; 15,94 \%)$ по сравнению с I типом $\left(\mathrm{n}=66 ; 33,5 \% ; \chi^{2}=18,856 ; \mathrm{df}=1\right.$; $\mathrm{p}=0,000)$. Результаты сравнения пациентов I и II типов по клиническим переменным представлены в таблице 3.

Т а б ли ц а 3 Сравнение групп пациентов с I и II типами алкогольной зависимости по клиническим переменным

\begin{tabular}{|l|c|c|c|}
\hline $\begin{array}{c}\text { Данные о развитии и течении } \\
\text { алкогольной зависимости }\end{array}$ & $\begin{array}{c}\text { I тип, me- } \\
\text { dian (IQR) } \\
\text { (n=197) }\end{array}$ & $\begin{array}{c}\text { II тип, me- } \\
\text { dian (IQR) } \\
\text { (n=251) }\end{array}$ & -value \\
\hline Возраст формирования СОА, лет & $37(33-43)$ & $26(24-30)$ & $\mathbf{0 , 0 0 0}$ \\
\hline $\begin{array}{l}\text { Срок формирования СОА от } \\
\text { возраста начала злоупотребления } \\
\text { алкоголем, лет }\end{array}$ & $5(2-7)$ & $5(3-7)$ & $\mathbf{0 , 0 1 2}$ \\
\hline ВАШ (при поступлении), баллов & $58(33-84)$ & $74(46-90)$ & $\mathbf{0 , 0 0 2}$ \\
\hline ВАШ (при выписке), баллов & $0(0-9)$ & $4(0-13)$ & $\mathbf{0 , 0 0 0}$ \\
\hline ВАШ (дельта), баллов & $49(27-78)$ & $57(36-79)$ & $\mathbf{0 , 0 2 6}$ \\
\hline
\end{tabular}
\begin{tabular}{|l|c|c|c|}
\hline ВАШ (дельта), баллов & $49(27-78)$ & $57(36-79)$ & $\mathbf{0 , 0 2 6}$ \\
\hline
\end{tabular}

П р и м е ч а н и е. Статистически значимые различия $(\mathrm{p}<0,05)$ выделены жирным.

Синдром отмены алкоголя (СОА) формировался достоверно раньше у пациентов с II типом (median $(\mathrm{IQR})=26$ (24-30) лет), чем у пациентов с I типом (median $(\mathrm{IQR})=37$ (33-43) лет, $\mathrm{p}=0,000)$. Срок формирования COA был достоверно меньше у пациентов с I типом (median $(\mathrm{IQR})=5$ (2-7) лет, чем у пациентов с II типом (median (IQR)=5 (3-7) лет, $\mathrm{p}=0,012)$. 
Тяга к алкоголю (ВАШ) при поступлении была достоверно выше у пациентов с II типом (median $(\mathrm{IQR})=74$ (46-90), чем у пациентов с I типом (median $(\mathrm{IQR})=58(33-84) ; \mathrm{p}=0,002)$. При выписке тяга к алкоголю также была достоверно выше у пациентов с II типом (median $(\mathrm{IQR})=4 \quad(0-13)$, чем у пациентов c I типом (median (IQR)=0 (0-9); p=0,000). Динамика уменьшения тяги к алкоголю была выше у пациентов c II типом (median (IQR)=57 (36-79), чем у пациентов с I типом (median $(\mathrm{IQR})=49$ (27-78); $\mathrm{p}=0,026)$.

Таким образом, у пациентов с условно наследственным типом АЗ СОА формируется дольше, чем у пациентов с I типом, несмотря на более ранний возраст формирования синдрома отмены. Тяга к алкоголю у пациентов с II типом АЗ достоверно выше как при поступлении, так и при выписке.

Результаты сравнения пациентов I и II типов по сумме случаев $\mathrm{CO}$, наличию и отсутствию $\mathrm{CO}$, степени СО представлены в таблице 4.

Т а б ли ц а 4

Сравнение групп пациентов с I и II типами алкогольной зависимости по семейной отягощенности

\begin{tabular}{|l|c|c|c|}
\hline $\begin{array}{c}\text { Данные о развитии и течении } \\
\text { алкогольной зависимости }\end{array}$ & $\begin{array}{c}\text { I тип, } \\
\text { mean } \pm \text { SE/\% } \\
(\mathrm{n}=197)\end{array}$ & $\begin{array}{c}\text { II тип, } \\
\text { mean } \pm \text { SE/\% } \\
(\mathrm{n}=251)\end{array}$ & $\begin{array}{c}\mathrm{p}- \\
\text { value }\end{array}$ \\
\hline Сумма случаев СО & $1,15 \pm 0,59$ & $1,47 \pm 0,69$ & $\mathbf{0 , 0 0 2}$ \\
\hline Доля пациентов с наличием СО & $\begin{array}{c}79,69 \% \\
(\mathrm{n}=157)\end{array}$ & $\begin{array}{c}88,04 \% \\
(\mathrm{n}=221)\end{array}$ & $\mathbf{0 , 0 1 6}$ \\
\hline $\begin{array}{l}\text { Доля пациентов со средней сте- } \\
\text { пенью СО }\end{array}$ & $\begin{array}{c}52,28 \% \\
(\mathrm{n}=103)\end{array}$ & $\begin{array}{c}49 \% \\
(\mathrm{n}=123)\end{array}$ & 0,272 \\
\hline Доля пациентов с высокой сте- & $\begin{array}{c}25,19 \% \\
\mathrm{n}=54)\end{array}$ & $\begin{array}{c}37,91 \% \\
(\mathrm{n}=98)\end{array}$ & $\mathbf{0 , 0 0 7}$ \\
\hline пенью СО & $\mathrm{n}$ & \\
\hline
\end{tabular}

П р и м е ч а н и е. Статистически значимые различия ( $<<0,05)$ выделены жирным.

В среднем число случаев АЗ в семье достоверно выше у пациентов II типа (mean $\pm \mathrm{SE}=1,47 \pm 0,69)$, чем у пациентов I типа (mean $\pm \mathrm{SE}=1,15 \pm 0,59 ; \mathrm{p}=0,002)$. Доля пациентов с наличием CO достоверно выше у пациентов II типа (88,04\%), чем у пациентов I типа $\left(79,69 \% ; \chi^{2}=5,84 ; \mathrm{df}=1 ; \mathrm{p}=0,016\right)$. Доля пациентов с высокой степенью СО достоверно больше у пациентов II типа $(37,91 \%)$, чем у пациентов I типа $\left(25,19 \% ; \chi^{2}=9,173 ; \mathrm{df}=2 ; \mathrm{p}=0,007\right)$. Таким образом, семейная отягощенность по сумме случаев и по доле пациентов с ее наличием и высокой степенью выше у пациентов с условно наследственным типом АЗ.

При сравнении пациентов по доле пациентов с высокой степенью генетического риска достоверных различий выявлено не было (табл. 5).

Т а б ли ц а 5

Сравнение групп пациентов с I и II типами алкоголь-
ной зависимости по генетическому риску
\begin{tabular}{|l|c|c|c|}
\hline $\begin{array}{c}\text { Данные о развитии и течении } \\
\text { алкогольной зависимости }\end{array}$ & $\begin{array}{c}\text { I тип, \% } \\
(\mathrm{n}=197)\end{array}$ & $\begin{array}{c}\text { II тип, \% } \\
(\mathrm{n}=251)\end{array}$ & $\begin{array}{c}\mathrm{p}- \\
\text { value }\end{array}$ \\
\hline $\begin{array}{l}\text { Доля пациентов с высоким } \\
\text { ГР, баллов }\end{array}$ & $44,16 \%(\mathrm{n}=87)$ & $49 \%(\mathrm{n}=123)$ & 0,308 \\
\hline
\end{tabular}

В общей группе сравнения, несмотря на то что доля пациентов с высоким ГР была больше у пациентов с II типом (49\%), чем у пациентов с I типом $(44,16 \%)$, достоверных различий не выявлено. Таким образом, уровень высокого генетического риска не отличается у пациентов I и II типов.
II. Сравнение групп пациентов мужского пола с I и II типами алкогольной зависимости

Пациенты мужского пола с I и II типами А3 сравнивались по данным о развитии и течении алкогольной зависимости. В нашей выборке 131 пациент $(38,3 \%)$ были I типа, 211 пациентов $(61,7 \%)$ - II типа. Результаты сравнения пациентов I и II типов по клиническим переменным показаны в таблице 6.

Т а б л и ц а 6

Сравнение групп пациентов мужского пола с I и II типами алкогольной зависимости по клиническим переменным

Данные о развитии и течении алкогольной зависимости $\quad$ an (IQR) $\quad$ an (IQR)

\begin{tabular}{l|c|c|c|} 
& $(\mathrm{n}=131)$ & $(\mathrm{n}=211)$ & \\
\hline Возраст формирования СОА, лет & $35(33-42)$ & $26(24-30)$ & $\mathbf{0 , 0 0 0}$ \\
\hline
\end{tabular} \begin{tabular}{|l|c|c|c|}
\hline Срок формирования СОА от & $5(2-7)$ & $5(3-7)$ & 0,135
\end{tabular} возраста начала злоупотребления алкоголем, лет

\begin{tabular}{|l|c|c|c|}
\hline ВАШ (при поступлении), баллов & 62 (38-85) & 74 (46-92) & $\mathbf{0 , 0 3 0}$ \\
\hline
\end{tabular}

\begin{tabular}{|l|c|c|c|}
\hline ВАШ (при выписке), баллов & $0(0-9)$ & $4(0-14)$ & $\mathbf{0 , 0 0 0}$ \\
\hline
\end{tabular}

\begin{tabular}{|l|c|c|c|}
\hline ВАШ (дельта), баллов & 50 (30-78) & 57 (37-80) & 0,163 \\
\hline
\end{tabular}

П р и м е ч а н и е. Статистически значимые различия $(p<0,05)$ выделены жирным.

COА формировался достоверно раньше у пациентов с II типом (median (IQR)=26 (24-30) лет), чем у пациентов с I типом (median $(\mathrm{IQR})=35$ (33-42) лет, $\mathrm{p}=0,000)$. Тяга к алкоголю при поступлении была достоверно выше у пациентов с II типом (median $(\mathrm{IQR})=74$ (46-92), чем у пациентов с I типом (median $(\mathrm{IQR})=62$ (38-85); p=0,03). При выписке тяга к алкоголю была также достоверно выше у пациентов с II типом (median $(\mathrm{IQR})=4(0-14)$, чем у пациентов с I типом (median $(\mathrm{IQR})=0(0-9) ; \mathrm{p}=0,000)$.

Таким образом, у пациентов мужского пола с условно наследственным типом АЗ СОА формируется раньше, чем у пациентов с I типом. При этом срок формирования СОА, в отличие от результатов, полученных в общей группе, достоверно не различается. Тяга к алкоголю у пациентов мужского пола c II типом А3 достоверно выше как при поступлении, так и при выписке.

Результаты сравнения пациентов мужского пола I и II типов по сумме случаев CO, наличию и отсутствию СО, степени СО представлены в таблице 7.

Т а б ли ц а 7

Сравнение групп пациентов мужского пола с I и II типами алкогольной зависимости по семейной отягощенности \begin{tabular}{|l|l|l|c|}
\hline Данные о развитии и течении & I тип, mean & II тип, mean & p-
\end{tabular}

\begin{tabular}{|l|c|c|c|}
\hline алкогольной зависимости & $\pm \mathrm{SE} / \%$ & $\pm \mathrm{SE} / \%$ & value \\
& $(\mathrm{n}=131)$ & $(\mathrm{n}=211)$ & \\
\hline
\end{tabular}

\begin{tabular}{|l|c|c|c|}
\hline Сумма случаев CO & $1,1 \pm 0,67$ & $1,43 \pm 0,68$ & $\mathbf{0 , 0 0 4}$ \\
\hline
\end{tabular}

\begin{tabular}{|l|c|c|c|}
\hline Доля пациентов с наличием СО & $79,38 \% ;$ & $88,62 \%$ & $\mathbf{0 , 0 2 0}$ \\
\hline
\end{tabular}

\begin{tabular}{|l|c|c|c|}
\hline Доля пациентов со средней сте- & $\begin{array}{c}54,19 \% \\
(\mathrm{n}=104)\end{array}$ & $\begin{array}{c}58,71 \% \\
(\mathrm{n}=187)\end{array}$ & 0,290 \\
пенью СО & $\mathrm{n}=107)$ & \\
\hline $\begin{array}{l}\text { Доля пациентов с высокой сте- } \\
\text { пенью СО }\end{array}$ & $\begin{array}{c}27,41 \% \\
(\mathrm{n}=33)\end{array}$ & $\begin{array}{c}39,04 \% \\
(\mathrm{n}=80)\end{array}$ & $\mathbf{0 , 0 1 0}$ \\
\hline
\end{tabular}

П р и м е ч а н и е. Статистически значимые различия $(\mathrm{p}<0,05)$ выделены жирным.

В среднем число случаев АЗ в семье достоверно выше у пациентов II типа (mean $\pm \mathrm{SE}=1,43 \pm 0,68)$, чем у пациентов I типа (mean $\pm \mathrm{SE}=1,1 \pm 0,67 ; \mathrm{p}=0,004)$. Доля пациентов с наличием СО достоверно выше 
при II типе $(88,62 \%)$, чем у пациентов I типа $\left(79,38 \% ; \chi^{2}=5,434 ; \mathrm{df}=1 ; \mathrm{p}=0,02\right)$. Доля пациентов с высокой степенью СО достоверно больше у пациентов II типа $(39,04 \%)$, чем у пациентов I типа $\left(27,41 \% ; \chi^{2}=8,535 ; \mathrm{df}=2 ; \mathrm{p}=0,01\right)$.

Таким образом, семейная отягощенность как по сумме случаев, так и по доле пациентов с ее наличием и высокой степенью выше у пациентов мужского пола с условно наследственным типом АЗ.

При сравнении пациентов мужского пола по доле пациентов с высокой степенью генетического риска достоверных различий выявлено не было (табл. 8).

Т а б ли ц а 8

Сравнение групп пациентов мужского пола с I и II типами алкогольной зависимости генетическому риску

\begin{tabular}{|l|c|c|c|}
\hline $\begin{array}{c}\text { Данные о развитии и течении } \\
\text { алкогольной зависимости }\end{array}$ & $\begin{array}{c}\text { I тип, \% } \\
(\mathrm{n}=131)\end{array}$ & $\begin{array}{c}\text { II тип, \% } \\
\text { (n=211) }\end{array}$ & $\begin{array}{c}\mathrm{p}- \\
\text { value }\end{array}$ \\
\hline Доля пациентов с высоким & $\begin{array}{c}47,33 \% \\
(\mathrm{n}=62)\end{array}$ & $\begin{array}{c}50,71 \% \\
(\mathrm{n}=107)\end{array}$ & 0,601 \\
ГР, баллов & \\
\hline
\end{tabular}

Таким образом, уровень высокого генетического риска не отличается у пациентов I и II типов мужского пола.

III. Сравнение групп пациентов женского пола с I и II типами алкогольной зависимости

Пациенты женского пола с I и II типами АЗ сравнивались по данным о развитии и течении алкогольной зависимости. В нашей выборке 66 пациенток $(62,3 \%)$ были I типа, 40 пациенток $(37,8 \%)$ - II типа. Результаты сравнения пациентов I и II типа по клиническим переменным представлены в таблице 9.

Т а б ли ц а 9

Сравнение групп пациентов женского пола с I и II типами алкогольной зависимости по клиническим переменным \begin{tabular}{|l|l|l|l|}
\hline Данные о развитии и течении & I тип, me- & II тип, me- & p-value \\
\hline
\end{tabular} \begin{tabular}{l|l|l} 
алкогольной зависимости & $\operatorname{dian}(\mathrm{IQR})$ & $\operatorname{dian}(\mathrm{IQR})$
\end{tabular} \begin{tabular}{l|ccc|} 
& $(\mathrm{n}=197)$ & $(\mathrm{n}=251)$ & \\
\hline Возраст формирования СОА, лет & $39(33-44)$ & $26(23-33)$ & $\mathbf{0 , 0 0 0}$ \\
\hline Срок формирования СОА от & $3(2-7)$ & $7(2-10)$ & $\mathbf{0 , 0 3 2}$
\end{tabular} \begin{tabular}{|l|c|c|c|}
$\begin{array}{l}\text { Срок формирования СОА от } \\
\text { возраста начала злоупотребления }\end{array}$ & $3(2-7)$ & $7(2-10)$ & $\mathbf{0 , 0 3 2}$ \\
\end{tabular} возраста начала злоголем, лет
алкогол

\begin{tabular}{|l|l|l|l|}
\hline ВАШ (при поступлении), баллов & 50 (25-80) & 74 (35-87) & 0,092 \\
\hline
\end{tabular}

\begin{tabular}{|l|c|c|c|}
\hline ВАШ (при выписке), баллов & $0(0-6)$ & $0(0-10)$ & 0,522 \\
\hline
\end{tabular}

\begin{tabular}{|l|c|c|c|}
\hline ВАШ (дельта), баллов & 46 (19-67) & 63 (31-76) & 0,122 \\
\hline
\end{tabular}

П р и м е ч а н и е. Статистически значимые различия $(\mathrm{p}<0,05)$ выделены жирным.

COA формировался достоверно раньше у пациентов с II типом (median $(\mathrm{IQR})=26$ (23-33) лет), чем у пациентов с I типом (median $(\mathrm{IQR})=39$ (33-44) лет, $\mathrm{p}=0,000)$. Срок формирования COA был достоверно меньше у пациентов с I типом (median $(\mathrm{IQR})=3(2-7)$ лет, чем у пациентов с II типом (median (IQR)=7 (210) лет, $\mathrm{p}=0,032)$.

Достоверных различий по выраженности тяги к алкоголю выявлено не было.

Таким образом, у пациентов женского пола с условно наследственным типом АЗ СОА формируется дольше, чем у пациентов с I типом, несмотря на более ранний возраст формирования синдрома отмены. При этом выраженность тяги к алкоголю как при поступлении, так и при выписке достоверно не различается.
Результаты сравнения пациентов женского пола I и II типов по сумме случаев семейной отягощенности, наличию и отсутствию СО, степени СО представлены в таблице 10.

Т а б л и ц а 10

Сравнение групп пациентов женского пола с I и II типами алкогольной зависимости по семейной отягощенности

Данные о развитии и течении I тип, mean \pm II тип, mean p-value

\begin{tabular}{l|c|c|c|}
\multicolumn{1}{c|}{ алкогольной зависимости } & $\begin{array}{c}\mathrm{SE} / \% \\
(\mathrm{n}=66)\end{array}$ & $\begin{array}{c} \pm \mathrm{SE} / \% \\
(\mathrm{n}=40)\end{array}$ & \\
\hline Сумма случаев СО & $1,18 \pm 0,1$ & $1,73 \pm 0,25$ & 0,103 \\
\hline Доля пациентов с наличием & $\begin{array}{c}80,3 \% \\
(\mathrm{n}=53)\end{array}$ & $\begin{array}{c}85 \% \\
(\mathrm{n}=34)\end{array}$ & 0,541 \\
СО & $\begin{array}{c}48,48 \% \\
(\mathrm{n}=32)\end{array}$ & $40 \%(\mathrm{n}=16)$ & 0,890 \\
$\begin{array}{l}\text { Доля пациентов со средней } \\
\text { степенью СО }\end{array}$ & $\begin{array}{c}31,81 \% \\
(\mathrm{n}=21)\end{array}$ & $45 \%(\mathrm{n}=18)$ & 0,290 \\
\hline $\begin{array}{l}\text { Доля пациентов с высокой } \\
\text { степенью СО }\end{array}$ & \multicolumn{3}{|l}{} \\
\hline
\end{tabular}

Достоверных различий по сумме случаев СО, наличию и отсутствию СО, степени СО в группе пациентов женского пола выявлено не было.

Таким образом, семейная отягощенность АЗ не отличается у женщин с I и II типами АЗ.

При сравнении пациентов женского пола по доле пациентов с высокой степенью генетического риска достоверных различий выявлено не было (табл. 11).

Т а б л и ц а 11

Сравнение групп пациентов женского пола с I и II типами алкогольной зависимости генетическому риску

\begin{tabular}{|l|c|c|c|}
\hline $\begin{array}{c}\text { Данные о развитии и течении } \\
\text { алкогольной зависимости }\end{array}$ & $\begin{array}{c}\text { I тип, \% } \\
(\mathrm{n}=66)\end{array}$ & $\begin{array}{c}\text { II тип, \% } \\
(\mathrm{n}=40)\end{array}$ & $\begin{array}{c}\mathrm{p}- \\
\text { value }\end{array}$ \\
\hline Доля пациентов с высоким & $37,87 \%$ & $45 \%$ & 0,469 \\
ГР, баллов & $(\mathrm{n}=25)$ & $(\mathrm{n}=18)$ & \\
\hline
\end{tabular}

ГР, баллов

Уровень высокого генетического риска не отличается у пациентов I и II типов женского пола.

\section{ВЫВОДЫ}

Алкогольная зависимость II типа по Клонинджеру с ранним началом злоупотребления встречается в 2 раза чаще у мужчин, чем у женщин, однако около $40 \%$ женщин относятся к этому типу. По результатам исследования II типу свойственны более длительный срок формирования синдрома отмены алкоголя, лучшая динамика уменьшения тяги к алкоголю за время госпитализации при её большей выраженности при поступлении, высокая доля пациентов с семейной отягощенностью, особенно её высокой степени, большее число случаев алкоголизма в семье. Это позволяет предположить, что II тип имеет высокий вклад наследственных факторов как в риск развития, так и в клинические проявления заболевания.

\section{КОНФЛИКТ ИНТЕРЕСОВ}

Авторы заявляют об отсутствии конфликта интересов в связи с публикацией данной статьи.

\section{ИСТОЧНИК ФИНАНСИРОВАНИЯ}

Авторы заявляют об отсутствии финансирования при проведении исследования.

\section{СООТВЕТСТВИЕ ПРИНЦИПАМ ЭТИКИ}

Работа соответствует этическим стандартам Хельсинкской Декларации ВМА и одобрена этическим комитетом Московского НИИ психиатрии филиал НМИЦПН им. В.П. Сербского. 


\section{ЛИТЕРАТУРА}

1. Анохина И.П. Наследственная предрасположенность к злоупотреблению психоактивными веществами. Психиатрия $u$ психофармакотерапия. 2001; 3: 59-65.

2. Гофман А.Г. Клиника алкогольного абстинентного синдрома. Вопросы наркологии. 2012; 6: 82-90.

3. Иващенко Д.В., Брюн Е.А., Савченко Л.М., Сычев Д.А Терапия неосложненного синдрома отмены алкоголя с позиций доказательной медицины: фокус на бензодиазепины. Наркология. 2016; 12: 83-91.

4. Кибитов А.О., Воскобоева Е.Ю., Бродянский В.М., Чупрова Н.А., Смирнова Е.В. Влияние фактора семейной отягощенности алкоголизмом и величины ее плотности на возрастные, динамические и качественные параметры формирования зависимости от алкоголя: опыт системного исследования. Наркология. 2010; 1: 59-70.

5. Кибитов А.О. ДНК-диагностика генетического риска развития наркологических заболеваний в рамках медикогенетического консультирования: основные принципы и опыт пилотного проекта. Вопросы наркологии. 2012; 5: 118-132.

6. Кибитов А.О. Генетические маркеры клинических вариантов развития наркологических заболеваний. Вопросы наркологии. 2013; 2: 131-146.

7. Кибитов А.О. Семейная отягощенность по наркологическим заболеваниям: биологические, клинические и генетические характеристики. Социальная и клиническая психиатрия. 2015; 25 (1): 98-104.

8. Кибитов А.О., Анохина И.П. Генетические основы этиологии и патогенеза болезней зависимости от психоактивных вешеств. Наркология. 2016; 15 (6): 84-104.

9. Николишин А.Е., Чупрова Н.А., Бродянский В.М., Щурина А.В., Сулимов Г.Ю., Ромашкин Р.А. Возможности прогноза темпа формирования и развития алкогольной зависимости: количественная оценка семейной отягощенности и уровень генетического риска. Практическая медицина. 2017; 2 (1): $62-65$.
10. Николишин А.Е., Чупрова Н.А., Ромашкин Р.А., Соловьева М.Г. Влияние генетических факторов на сроки формирования и развития алкогольной зависимости: семейная отягощенность и генетический риск. Вопросы наркологии. 2017; 1: 73-88.

11. Яковлев А.Н., Пашкевич Н.В., Пажитных Д.В., Ткачев А.А., Бродянский В.М., Чупрова Н.А., Щурина А.В., Ромашкин Р.А., Витчинкина В.И. Поиск генетических и психологических маркеров риска потребления наркотиков подростками с аддиктивным поведением в рамках первичной профилактики: предварительные результаты пилотного исследования в Липецке. Сибирский вестник психиатрии и наркологии. 2017; 2: 5-11.

12. Bienvenu O.J., Davydow D.S., Kendler K.S. Psychiatric 'diseases' versus behavioral disorders and degree of genetic influence. Psychological Medicine. 2011; 41: 33-40.

13. Cloninger C.R., Sigvardsson S., Bohman M. Type I and Type II Alcoholism: An Update. Alcohol Health \& Research World. 1996; 20 (1): 18-23.

14. Ducci F., Goldman D. Genetic approaches to addiction: genes and alcohol. Addiction. 2008: 103 (Iss. 9): 1414-1428.

15. Enoch M.A., Goldman D. The genetics of alcoholism and alcohol abuse. Current Psychiatry Reports. 2001; 3 (Iss. 2): 144 151

16. Stoltenberg S.F., Mudd S.A., Blow F.C., Hill E.M. Evaluating measures of family history of alcoholism: density versus dichotomy. Addiction. 1998; 93 (Iss. 10): 1511-1520.

17. Walters G.D. The heritability of alcohol abuse and dependence: a meta-analysis of behavior genetic research. The American Journal of Drug and Alcohol Abuse. 2002; 28 (Iss. 3): 557-584

Поступила в редакцию 12.02.2018 Утверждена к печати 2.04.2018

Николишин Антон Евгеньевич, научный сотрудник лаборатории молекулярной генетики.

Бродянский Вадим Маркович, к.б.н., ведущий научный сотрудник лаборатории молекулярной генетики. Чупрова Наталья Александровна, научный сотрудник лаборатории молекулярной генетики.

Сулимов Герман Юрьевич, к.м.н., заместитель директора Научно-исследовательский кластер АО "Врачи".

Кибитов Александр Олегович, д.м.н., руководитель лаборатории молекулярной генетики.

Николишин Антон Евгеньевич, aenikolishin@gmail.com

УДК 616.89-008.441.12:575.174.015.3

For citation: Nikolishin A.E., Brodyansky V.M., Chuprova N.A., Sulimov G.Yu, Kibitov A.O. In search for hereditary form of alcohol addiction: Cloninger's typology, dynamics of withdrawal formation, family history and evaluation of genetic risk. Siberian Herald of Psychiatry and Addiction Psychiatry. 2018; 2 (99): 82-88. https://doi.org/10.26617/1810-3111-2018-2(99)-82-88

\title{
In search for hereditary form of alcohol addiction: Cloninger's typology, dynamics of withdrawal formation, family history and evaluation of genetic risk
}

\author{
Nikolishin A.E. ${ }^{1}$, Brodyansky V.M. ${ }^{1}$, Chuprova N.A. ${ }^{1}$, Sulimov G.Yu. ${ }^{2}$, Kibitov A.O. \\ ${ }^{1}$ V.P. Serbsky National Medical Research Center of Psychiatry and Narcology \\ Kropotkinsky Lane 23, 119991, Moscow, Russian Federation \\ ${ }^{2}$ Research Cluster JSC "Vrachi" \\ Street Gabrichevsky, 5, 125367, Moscow, Russian Federation
}

ABSTRACT

Objective: comparative analysis of patients with type I and type II alcoholism (A) according to Cloninger with use of quantitative evaluations of family history (FH), clinical characteristics of development and course of A, level of individual genetic risk of development of A according to data of genomic profiling. Material and Methods. 464 inpatient, mean age $42.48 \pm 0.504$ years; diagnosis "Dependence Syndrome" (F10.2 according to ICD-10) were examined. Three comparisons between patients with type I and type II A were performed: in total group of patients, in group of male and female patients. Results. In total group of comparison in patients with type II A alcohol withdrawal syndrome (AWS) was formed longer, than in patients with type I, despite earlier age of AWS formation, craving for alcohol I was higher both at admission and discharge. FH was more loaded in patients with type II A. In male patients with type II A AWS was formed earlier, craving for alcohol was reliably higher both at admission and discharge, than in patients with type I. FH was more loaded in male patients with type II type A. In female patients with type II A AWS was 
formed longer, than in patients with type I, despite earlier age of AWS formation, severity of craving for alcohol and FH did not differ reliably. FH did not differ in women with type I and type II A. Level of genetic risk did not differ in patients with type I and type II A in all comparisons. Conclusion. Type II A according to Cloninger with early onset of abuse was found twice as frequently in men than in women, however, about $40 \%$ of women suffered from this type. According to results of the study for type II A longer term of AWS formation, better dynamics of reduction of craving for for alcohol are typical during hospitalization with greatest severity at admission, high portion of patients with FH, especially of its higher loading, larger number of cases of alcoholism in the family. It allowed assuming that type II had a high contribution of hereditary factors to risk of development and clinical implications of disease.

\section{Keywords: alcoholism, genetics, heredity, withdrawal syndrome, family history, genetic risk, gene polymorphism, dopamine.}

\section{REFERENCES}

1. Anokhina I.P. Nasledstvennaya predraspolozhennost k zloupotrebleniyu psihoaktivnyimi veschestvami [Hereditary predisposition to substance abuse]. Psihiatriya i psihofarmakoterapiya - Psychiatry and Psychopharmacotherapy. 2001; 3: 59-65 (in Russian).

2. Hoffman A.G. Klinika alkogolnogo abstinentnogo sindroma [Clinical picture of the alcohol withdrawal syndrome]. Voprosyi narkologii - Journal of Addiction Problems. 2012; 6: 82-90 (in Russian).

3. Ivashchenko D.V., Bryun E.A., Savchenko L.M., Sychev D.A. Terapiya neoslozhnennogo sindroma otmenyi alkogolya s pozitsiy dokazatelnoy meditsinyi: fokus na benzodiazepinyi [Evidence-based treatment of non-complicated alcohol withdrawal syndrome: focus on benzodiazepines tranquilizers]. Narkologiya - Narcology. 2016; 12: 83-91 (in Russian).

4. Kibitov A.O., Voskoboeva E.Yu., Brodyansky V.M., Chuprova N.A., Smirnova E.V. Vliyanie faktora semeynoy otyagoschennosti alkogolizmom i velichinyi ee plotnosti na vozrastnyie, dinamicheskie i kachestvennyie parametryi formirovaniya zavisimosti ot alkogolya: opyit sistemnogo issledovaniya [Positive family history of alcoholism and its density modify age, dynamic and qualitative parameters of alcohol dependence development: experience of system study]. Narkologiya - Narcology. 2010; 1: 59-70 (in Russian).

5. Kibitov A.O. DNK-diagnostika geneticheskogo riska razvitiya narkologicheskih zabolevaniy $\mathrm{v}$ ramkah mediko-geneticheskogo konsultirovaniya: osnovnyie printsipyi i opyit pilotnogo proekta [DNA diagnosis of level of genetic risk of substance abuse as part of genetic counseling: basic principles and the experience of the pilot project]. Voprosyi narkologii - Journal of Addiction Problems. 2012; 5: 118-132 (in Russian).

6. Kibitov A.O. Geneticheskie markeryi klinicheskih variantov razvitiya narkologicheskih zabolevaniy [Genetic markers of the clinical variants of substance dependence development]. Voprosyi narkologii - Journal of Addiction Problems. 2013; 2: 131-146 (in Russian).

7. Kibitov A.O. Semeynaya otyagoschennost po narkologicheskim zabolevaniyam: biologicheskie, klinicheskie i geneticheskie harakteristiki [Familial vulnerability for addictions: biological, genetic and clinical characteristics. Sotsialnaya i klinicheskaya psihiatriya - Social and Clinical Psychiatry. 2015; 25 (1): 98104 (in Russian).

8. Kibitov A.O., Anokhina I.P. Geneticheskie osnovyi etiologii patogeneza bolezney zavisimosti ot psihoaktivnyih veschestv [The genetic basis of etiology and pathogenesis of substance dependence]. Narkologiya - Narcology. 2016; 15 (6): 84-104 (in Russian).
9. Nikolishin A.E., Chuprova N.A., Brodyansky V.M., Shchurina A.V., Sulimov G.Yu., Romashkin R.A. Vozmozhnosti prognoza tempa formirovaniya i razvitiya alkogolnoy zavisimosti: kolichestvennaya otsenka semeynoy otyagoschennosti i uroven geneticheskogo riska [The ability to forecast the rate of alcohol dependence formation and development: quantitative analysis of family history and the level of genetic risk]. Prakticheskaya meditsina - Practical Medicine. 2017; 2 (1): 62-65 (in Russian).

10. Nikolishin A.E., Chuprova N.A., Romashkin R.A., Solovyeva M.G. Vliyanie geneticheskih faktorov na sroki formirovaniya $i$ razvitiya alkogolnoy zavisimosti: semeynaya otyagoschennost $\mathrm{i}$ geneticheskiy risk [The influence of genetic factors on the terms of alcohol dependence onset and development: loaded family history and genetic risk]. Voprosyi narkologii - Journal of Addiction Problems. 2017; 1: 73-88 (in Russian).

11. Yakovlev A.N., Pashkevich N.V., Pazhitnyih D.V., Tkachev A.A., Brodyanskiy V.M., Chuprova N.A., Schurina A.V., Romashkin R.A., Vitchinkina V.I. Poisk geneticheskih i psihologicheskih markerov riska potrebleniya narkotikov podrostkami $\mathrm{s}$ addiktivnyim povedeniem $\mathrm{v}$ ramkah pervichnoy profilak-tiki: predvaritelnyie rezultatyi pilotnogo issledovaniya $\mathrm{v}$ Lipetske [The search for genetic and psychological markers of the risk of drug use by adolescents with addictive behavior as part of primary prevention: preliminary results of a pilot study in Lipetsk]. Sibirskiy vestnik psihiatrii i narkologii - Siberian Herald of Psychiatry and Addiction Psychiatry. 2017; 2: 5-11 (in Russian).

12. Bienvenu O.J., Davydow D.S., Kendler K.S. Psychiatric 'diseases' versus behavioral disorders and degree of genetic influence. Psychological Medicine. 2011; 41: 33-40.

13. Cloninger C.R., Sigvardsson S., Bohman M. Type I and Type II Alcoholism: An Update. Alcohol Health \& Research World. 1996; 20 (1): 18-23.

14. Ducci F., Goldman D. Genetic approaches to addiction: genes and alcohol. Addiction. 2008: 103 (Iss. 9): 1414-1428.

15. Enoch M.A., Goldman D. The genetics of alcoholism and alcohol abuse. Current Psychiatry Reports. 2001; 3 (Iss. 2): 144151.

16. Stoltenberg S.F., Mudd S.A., Blow F.C., Hill E.M. Evaluating measures of family history of alcoholism: density versus dichotomy. Addiction. 1998; 93 (Iss. 10): 1511-1520.

17. Walters G.D. The heritability of alcohol abuse and dependence: a meta-analysis of behavior genetic research. The American Journal of Drug and Alcohol Abuse. 2002; 28 (Iss. 3): 557-584.

Received February 12.2018

Accepted April 2.2018

Nikolishin Anton E., researcher assistant of the Laboratory of Molecular Genetics, V.P. Serbsky National Medical Research Center of Psychiatry and Narcology, Moscow, Russian Federation.

Brodyansky Vadim M., PhD, Biology, lead researcher of the Laboratory of Molecular Genetics, V.P. Serbsky National Medical Research Center of Psychiatry and Narcology, Moscow, Russian Federation.

Chuprova Natalia A., researcher of the Laboratory of Molecular Genetics, V.P. Serbsky National Medical Research Center of Psychiatry and Narcology, Moscow, Russian Federation.

Sulimov German Yu., PhD, deputy director of Research Cluster JSC “Vrachi”, Moscow, Russian Federation.

Kibitov Alexander O., MD, Head of the Laboratory of Molecular Genetics, V.P. Serbsky National Medical Research Center of Psychiatry and Narcology, Moscow, Russian Federation.

Nikolishin Anton E., aenikolishin@gmail.com 